\title{
The Crystal and Molecular Structure of 2,5-Dihydroxy-
}

\section{1,4-benzoquinone at $-162{ }^{\circ} \mathrm{C}$}

\author{
DAG SEMMINGSEN
}

Department of Chemistry, University of Oslo, Oslo 3, Norway

The crystal structure of 2,5-dihydroxy-1,4benzoquinone has been determined at $-162^{\circ} \mathrm{C}$ from three-dimensional counter data. The space-group is $C 2 / c$ with $a=7.801(1), b=$ $6.951(1), c=9.939(2) \AA$, and $\beta=90.04^{\circ}$. Full matrix least-squares refinements have yielded a conventional $R$ value of 0.035 for $916 \mathrm{ob}$ servations having $\sin \theta / \lambda>0.55$. The molecules are situated at the centers of inversion, and there are two different $\mathrm{C}-\mathrm{O}$ bonds [1.235(1) and 1.327(1) $\AA$ ] and three different $\mathrm{C}-\mathrm{C}$ bonds $[1.506(1), 1.442(1)$ and $1.355(1) \AA]$. The planar structure shows some quinoid character although the double bonds are only partially localized. The hydrogen atom attached to the hydroxyl group appears to participate in two weak hydrogen bonds, one of which ties the molecules into infinite planar sheets, the other is a weak intramolecular interaction.

The crystal structure of the dipotassium salt of 2,5-dihydroxy-1,4-benzoquinone has recently been reported.' The anion $\mathrm{C}_{6} \mathrm{H}_{2} \mathrm{O}_{4}^{2-}$ was found to have $D_{2 h}$ symmetry in the crystal within experimental error, thus there is virtually complete delocalization of the $\pi$ electrons along the 1-oxopropen-3-olat fragment. Intermediate character although not complete conjugation in corresponding bonds has also been found in the crystal structures of fluoro- and chloroanilic acids. ${ }^{2,3}$ To further investigate the effects of conjugation in this type of molecule, the crystal structure of 2,5-dihydroxybenzoquinone was determined. The determination was also carried out as part of a series of structure investigations of compounds containing the 1-oxo-propen-3-ol chain.4,5

Acta Chem. Scand. B 31 (1977) No. 1

\section{EXPERIMENTAL}

The compound was synthesized by the method given by Jones and Shonle (Dec. 217 $\left.{ }^{\circ} \mathrm{C}\right),{ }^{\circ}$ and suitable deep-red to orange crystals were grown by slow cooling of a solution of the compound in absolute alcohol. The crystals were frequently found to be twinned with (101) as the twin plane. Oscillation and Weissenberg photographs indicated monoclinic symmetry. $(a=7.89, b=6.95, c=10.10 \AA, \beta=$ $\left.91.4^{\circ}, t=25^{\circ} \mathrm{C}\right)$. Systematic absence of reflections $h k l$ for $h+k=2 n+1$ and $h 0 l$ with $l$ odd determined the space group to be either $C c$ or $C 2 / c$. The latter was assumed and proved to be correct by the structure refinements. Cell dimensions at $-162^{\circ} \mathrm{C}$ were determined from the setting angles of 30 reflections with $2 \theta$ values larger than $\mathbf{3 5}^{\circ}$. Three-dimensional intensity data were collected from an almost rectangular crystal of dimensions $0.35 \times 0.45 \times$ $0.30 \mathrm{~mm}$ on a SYNTEX $\mathrm{P} \overline{\mathbf{l}}$ diffractometer with graphite monochromated MoK$\alpha$ radiation $\left(K \alpha_{1}, \lambda=0.70926 \AA ; K \alpha_{2}, \lambda=0.71354 \AA\right)$. The diffractometer was operated in the $\omega-2 \theta$ scanning mode and equipped with an Enraf-Nonius liquid nitrogen cooling device (modified by H. Hope). The temperature at the position of the crystal was $-162 \pm 2{ }^{\circ} \mathrm{C}$. Data were collected in one quadrant of reciprocal space to $2 \theta_{\max }=80^{\circ}$ with a variable scan speed dependent on intensity. Below $2 \theta=50^{\circ}$ the scan speed was varied between 2 and $8^{\circ} \min ^{-1}$, above $2 \theta=50^{\circ}$ between 2 and $4^{\circ} \mathrm{min}^{-1}$. In the latter shell a threshold value was applied to avoid measurement of very weak reflections $(<2 \sigma(I))$. A symmetric scan range of $2^{\circ}$ corrected for angular dependence of spectral dispersion were performed, the ratio of background time to scan time was 0.6. A total of 1406 intensities excluding space group absences were measured, of which 1294 were greater than $2 \sigma(I)$ and regarded as observed, the remaining 112 were rejected from the final structure analysis. The intensities and their standard deviations were corrected for Lorentz 
Table 1. Fractional coordinates and thermal parameters with estimated standard deviations: A, for the refinement with an inner cut-off at $\sin \theta / \lambda=0.55 \AA^{-1}, B$, for the refinement of all data. Expression for anisotropic vibration is $\exp \left[-2 \pi^{2}\left(h^{2} a^{* 2} U 11+\cdots+2 k l b^{*} c^{*} U 23\right)\right]$.
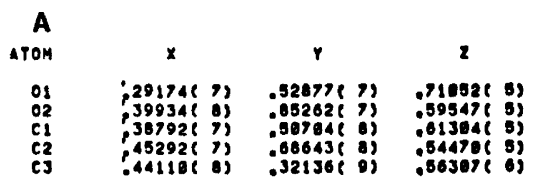

UIS

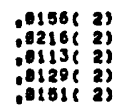

422
$.0131(2)$
$.01989(2)$
$00082(2)$
$.0092(2)$
$0000(2)$
$0003(2)$

ง33

U12

.0122( 2) .0008( 1)

$0168(2)$
$0099(2)$

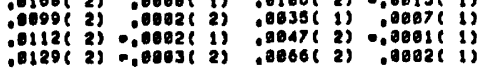

422

433

112

112

บ23

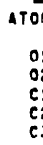

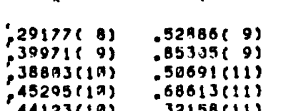

$.71041(6)$
$.59537(6)$
$.61288(?)$
$.54469(?)$
$.56331(9)$

U11

. $0138(2)$

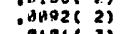

. $9191\left(\begin{array}{l}3) \\ . \operatorname{sog}(3)\end{array}\right.$

$.54469($ ?) $.0132(3)$,

inges 3$)$

.0130( 2) .001日e 2) .on75e 2) . Anuse 2)

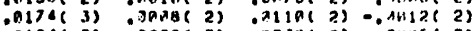

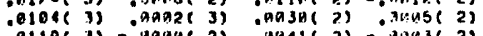

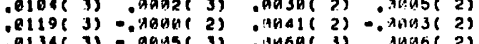

$\begin{array}{cc}2 & 8 \\ .0622(17) & 3.4(3)\end{array}$

Arom

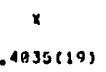

$r$
$.2197(21)$

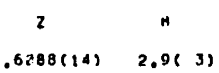

and polarization effects and a $2 \%$ uncertainty to account for experimental uncertainties was included in the latter. Atomic form factors used were those of Doyle and Turner ${ }^{7}$ for oxygen and carbon atoms and those of Stewart et $a l .^{8}$ for hydrogen atoms.

Computer programs employed during the present study are those of Refs. 9 and 10.

\section{CRYSTAL DATA $\left(-162^{\circ} \mathrm{C}\right)$}

2,5-Dihydroxy-1,4-benzoquinone, $\mathrm{C}_{6} \mathrm{H}_{4} \mathrm{O}_{4}$, monoclinic. Space group $C 2 / c, a=7.8008(14)$, $b=6.9405(13), c=9.987(19) \quad \AA, \beta=90.044(10)$, $V=538.1 \AA^{2}, M=140.10 \mathrm{~g} \mathrm{~mol}^{-1}, F_{(000)}=288$, $Z=4, D_{\text {calc }}=1.729 \mathrm{~g} \mathrm{~cm}^{-3}, D_{\text {obs }}\left(25^{\circ} \mathrm{C}\right)=1.65$ $\mathrm{g} \mathrm{\textrm {cm } ^ { - 8 }}$.

\section{SOLUTION AND REFINEMENTS}

The structure was solved by direct methods' and further refined by full matrix least squares methods. The function minimized was $\Sigma w$. $\left(F_{\mathrm{o}}-F_{\mathrm{c}}\right)^{2}, \quad w=\sigma\left(F_{\mathrm{o}}\right)^{-2}$. The hydrogen atoms were located in a difference Fourier map at the end of the isotropic refinement. However, the hydrogen atom $\left(\mathrm{H}_{\mathrm{O}}\right)$ attached to the hydroxylic oxygen was initially placed on the line joining the donor and acceptor of the inter. molecular hydrogen bond. During the subsequent refinements the isotropic temperature factor of $\mathrm{H}_{\mathrm{O}}$ increased from 3.0 to $5.5 \AA^{2}$, while the atom position shifted near to that determined from the difference map, whereupon the value of the thermal parameter again declined to $3.4 \AA^{2}$. Refinement with all data terminated with the residuals $R=0.036, R_{\mathrm{w}}=$ 0.044 and $S=2.16$. $^{*}$ In the final refinements only data for which $\sin \theta / \lambda>0.55$ were included, leaving 916 structure factors for a refinement of heavy atoms only. During these refinements the positional parameters shifted by up to 7 standard deviations, while all $U_{\text {ii }}$ were reduced by amounts up to 4 standard deviations. The residuals obtained were $R=$ $0.030, R_{\mathrm{w}}=0.030$ and $S=1.2$. Positional and thermal parameters from the refinement of "high" angle data are given in Table 1 together with the values from the refinement of all data.

In the following discussion heavy atom parameters from the high angle refinement will be considered, though only small changes in bond lengths (maximum $3 \times 10^{-3} \AA$ ) were observed.

An analysis of the molecular motion in terms of rigid body vibrations indicated that the molecule may be regarded as rigid since $\Delta U_{\mathrm{rms}}=$ $0.0003 \AA^{2.11,12}$ Corrections for librational motions range from $1 \times 10^{-3}(\mathrm{Cl}-\mathrm{Ol})$ to $4 \times 10^{-8}$ $\AA$ (Cl-C2) and have not been applied. The r.m.s. amplitudes range from 0.173 to $0.078 \AA$ with the larger component perpendicular to the molecular plane (see Fig. 1). The structure factors can be obtained from the author upon request.

\footnotetext{
* Standard deviations of unit weight $S=$ $\left[\sum W\left(F_{0}-F_{c}\right)^{2} /(m-n)\right]$.
} 


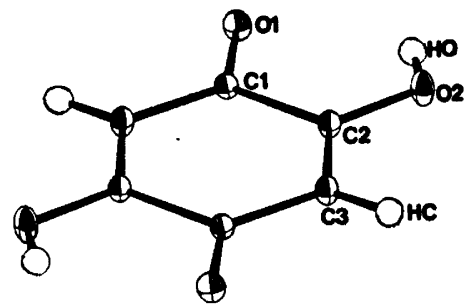

Fig. 1. Perspective drawing of the molecule with atom numbering as indicated. The shapes of the heavy atoms represent $50 \%$ probability contours of thermal motion.

\section{DESCRIPTION AND DISCUSSION}

The molecular structure of 2,5-dihydroxy-1,4benzoquinone in the crystalline state is shown in Fig. $1,{ }^{18}$ which also shows the atom numbering scheme. The molecules are situated at crystallographic centers of inversion. Bond lengths and angles from both refinements are given in Table 2 together with hydrogen bond parameters.

The molecular structure of 2,5-dihydroxy1,4-benzoquinone corresponds to that found previously in the chloro- and fluoroanilic acids., ${ }^{2,3}$ The structure is basically of quinoid character, however, some degree of conjuga-

Table 2. Interatomic distances, bond angles and hydrogen bond parameters, with estimated standard deviations: $A$, for the refinement with an inner cut off at $\sin \theta / \lambda=0.55 \AA^{-1}, B$, for the refinement of all data.

\begin{tabular}{|c|c|c|c|c|c|}
\hline $\begin{array}{l}\text { A } \\
\text { OIstance }\end{array}$ & (i) & & DISTANCE & (i) & \\
\hline 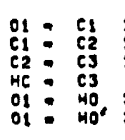 & $\begin{array}{l}1.2350 \\
1,95864 \\
1,3547 \\
09392 \\
2,2273 \\
2.1109\end{array}$ & $\left(\begin{array}{l}0 \\
\dddot{3} \\
3\end{array}\right.$ & $\begin{array}{l}0_{2}: c_{2} \\
c_{1}: c_{3} \\
\text { Mo: } 02 \\
01: 02 \\
01: 02 \\
01: 02\end{array}$ & $\begin{array}{l}1,32671 \\
1.44231 \\
.7987 \\
2.65838 \\
2,7275\end{array}$ & \\
\hline ANGLE & & $(0)$ & ANGLE & & $(\bullet)$ \\
\hline 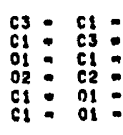 & $\begin{array}{l}\mathrm{C2} \\
\mathrm{C2} \\
\mathrm{C3} \\
\mathrm{C3} \\
\mathrm{HO} \\
\mathrm{HO}\end{array}$ & 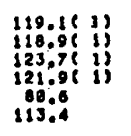 & $\begin{array}{l}c_{1}: c_{2}: \\
01: c_{1}: \\
02: c_{2}: \\
c_{2}:: 2 \\
02:{ }^{10}: \\
02\end{array}$ & $\begin{array}{l}\text { cg } \\
\text { c2 } \\
61 \\
\text { C1 } \\
\text { MO } \\
01 \\
08\end{array}$ & 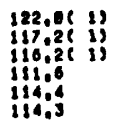 \\
\hline $\begin{array}{l}\text { B } \\
\text { DigtanCE }\end{array}$ & & i) & OISTANCE & is & is \\
\hline 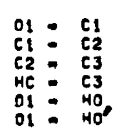 & $\begin{array}{l}1.2360 \\
1.5045 \\
1.3533 \\
.9426 \\
2.2296 \\
2.1106\end{array}$ & $\begin{array}{l}0(8) \\
\text { s(19) } \\
3(19) \\
(15) \\
(16) \\
(17)\end{array}$ & 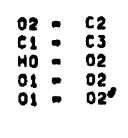 & $\begin{array}{l}1,3300 \\
1,4399 \\
2.8041 \\
2,6618 \\
2,7296\end{array}$ & 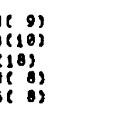 \\
\hline ANGLE & & $(0)$ & ANGLE & & $(\bullet)$ \\
\hline 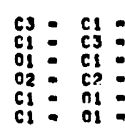 & $\begin{array}{l}c 2 \\
c 2 \\
c 3 \\
c 3 \\
c 3 \\
40 \\
40 \\
40\end{array}$ & $\begin{array}{c}119.18(1) \\
118.99(1) \\
123.78(1) \\
121.78(1) \\
89.88(5) \\
113.04(2)\end{array}$ & 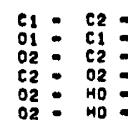 & 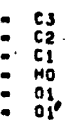 & $\begin{array}{l}222.01 \\
117.81 \\
116.11 \\
1111 \\
111.5111 \\
114.1114 \\
114.2(12)\end{array}$ \\
\hline
\end{tabular}

Acta Chem. Scand. B 31 (1977) No. 1 tion exists along the sequence of atoms $\mathrm{Ol}^{*}-$ C1*-C3-C2-O2. † Thus C1*-C3 [1.442(1) $\AA]$ is definitely shorter than $\mathrm{Cl}-\mathrm{C} 2$ [1.506(1) $\AA]$, indicating only minor conjugation across the latter bond. There is therefore markedly less conjugation in the 1-oxo-propen-3-ol chain than in the 1-oxo-propen-3-olat chain in the free acid and in the dipotassium salt. 1 The $\mathrm{Cl}-\mathrm{C} 2$ bond in the present study is also probably shorter than the corresponding value in the salt $[1.543(7) \AA]$. The bond lengths in the conjugated chain are similar to those found in the trans-enol arrangements of dimedone ${ }^{4}$ and phenylmalondialdehyde. ${ }^{5}$

A slight tendency towards higher conjugation in the $\mathrm{O} 1-\mathrm{Cl}-\mathrm{C} 3-\mathrm{C} 2-\mathrm{O} 2$ skeleton upon substitution of hydrogen for the more electron withdrawing halogens may be noted. The C-O double bond increases from 1.215(3) $\AA$ in fluoroanilic acid ${ }^{2}$ to 1.235 (1) $\AA$ in the present work, while the $\mathrm{C}-\mathrm{O}$ single bond decreases from 1.334(3) to 1.327 (1) $\AA$. Further the $\mathrm{C}-\mathrm{C}$ double bond increases from $1.332(4)$ to $1.355(1) \AA$, while the $\mathrm{Cl}-\mathrm{C} 3$ single bond contracts from $1.456(3)$ to $1.442(1) \AA$. On the other hand the slight expansion of the $\mathrm{Cl}-\mathrm{C} 2$ bond from $1.496(4)$ to $1.506(1) \AA$ may indicate a decrease in conjugation across this bond. The distances found in chloroanilic acid 3 lie between those found for fluoroanilic acid ${ }^{2}$ and the title com. pound except that the $\mathrm{C}-\mathrm{O}$ single bond length does not differ significantly from that found in the present work.

The molecule is planar within the estimated standard deviations. The average absolute discrepancy from the root-mean-square plane through the heavy atoms in the asymmetric unit is only $0.001 \AA$, and the largest deviation (0.002 $\AA$ for $\mathrm{Cl}$ ) is not significant. The hydrogen atoms also lie in this plane with deviations for $\mathrm{H}_{\mathrm{C}}$ and $\mathrm{H}_{\mathrm{O}}$ of 0.035 and $0.026 \AA$, respectively.

A packing diagram of the structure is shown in Fig. 2.13 The molecules are linked together to form infinite sheets of hydrogen bonded layers [0...O, 2.728(1) $\AA]$ extending in the (101) plane. The stacking of layers perpendicular to (101) is such that $\mathrm{C}-\mathrm{O}$ bonds of adjacent molecules overlap in an approximately antiparallel manner with an average layer separation of $3.16 \AA$. This suggests that the attractive

$\dagger$ Atoms marked with an asterisk are those centrosymmetrically related within the molecule. 

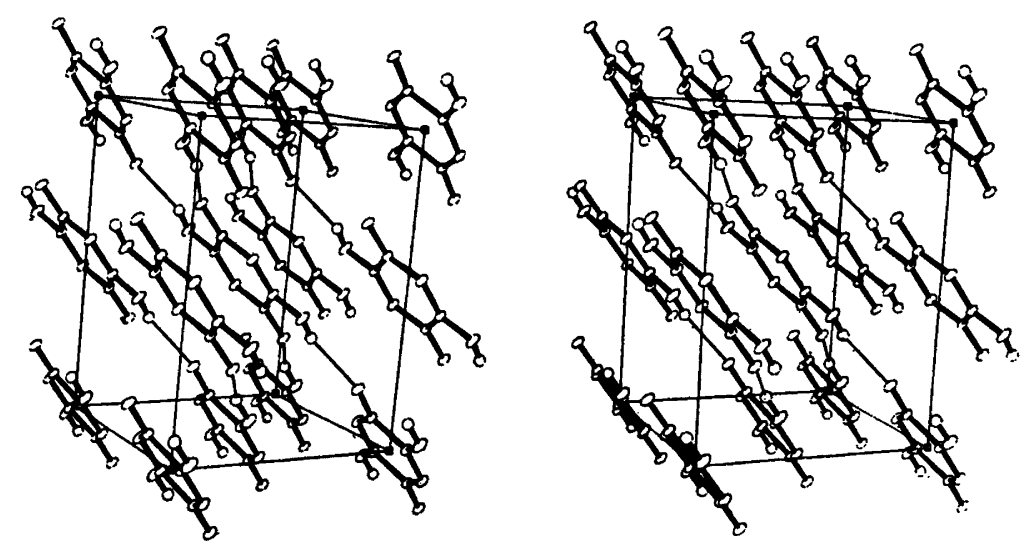

Fig. 2. Stereoscopic illustration of the crystal structure of 2,5-dihydroxy-1,4-benzoquinone. Thermal ellipsoids are scaled to include $50 \%$ probability.

forces between layers may be partly due to dipole-dipole interactions. ${ }^{14}$ The intermolecular contacts between these atoms do, however, correspond to normal van der Waals distances $(\mathrm{C} 1 \ldots \mathrm{O} 1,3.06 \AA ; \mathrm{C} 2 \ldots . .02 ; 3.09 \AA) .^{15}$ In addition to the 0 .... 0 hydrogen bonds there are two other intermolecular interactions within the sheets. These are from $\mathrm{H}_{\mathrm{C}}$ to $\mathrm{Ol}$ and $\mathrm{O2}$ of lengths 2.67 and $2.49 \AA$, respectively, and are similar to $\mathrm{CH}$-... $\mathrm{O}$ contacts commonly occurring in quinoid compounds. ${ }^{14}$

The intermolecular hydrogen bond [O-..-O, $2.728(1) \AA]$, is weak and of a similar length to those found in fluoro- and chloroanilic acids (2.720 and $2.769 \AA$, respectively)., ${ }^{2,3}$ The hydrogen atom is displaced significantly off the line joining the two oxygen atoms $(\angle \mathrm{O}-\mathrm{H} \cdots \mathrm{O}$ $=114^{\circ}$ ) with an $\mathrm{H}_{\mathrm{O}} \cdots \mathrm{O}$ separation of $2.12(2)$ A. An examination of intramolecular contacts reveals that the $\mathrm{H}_{\mathrm{O}}$-..Ol distance within the molecule is only $2.23(2) \AA$, and although longer than the intermolecular contact, it is shorter than the sum of van der Waals radii for hydrogen $(1.0 \AA)^{16}$ and oxygen $(1.4 \AA),{ }^{15}$ sug. gesting the presence of a bifuracted hydrogen bond arrangement. The corresponding intramolecular 0 ... O distance is 2.658(1) $\AA$ and the $\mathrm{O}-\mathrm{H} \ldots \mathrm{O}$ angles $\mathrm{i} 114^{\circ}$. The intramolecular interaction constituting a five-membered ring system is certainly weak, however somewhat similar arrangements have previously been found in the crystal structure of tropolone ${ }^{17}$ and there is also evidence of such interaction in (-). noradrenaline and some related compounds. ${ }^{18}$

\section{REFERENCES}

1. Kulpe, S. J. Prakt. Chem. 316 (1974) 353.

2. Krogh Andersen, E. and Krogh Andersen, I. G. Acta Crystallogr. $B 31$ (1975) 384.

3. Krogh Andersen, E. Acta Crystallogr. 22 (1967) 188.

4. Semmingsen, D. Acta Chem. Scand. B 28 (1974) 169.

5. Semmingsen, D. Acta Chem. Scand. B 31 (1977). In press.

6. Jones, R. G. and Shonle, H. A. J. Am. Chem. Soc. 67 (1945) 1034.

7. Doyle, P. A. and Turner, P. S. Acta Crystallogr. A 24 (1968) 390.

8. Stewart, R. F., Davidson, E. R. and Simpson, W. T. J. Chem. Phys. 42 (1965) 3175.

9. Germain, G., Main, P. and Woolfson, M. M. Acta Crystallogr. A 27 (1971) 368.

10. Groth, P. Acta Chem. Scand. 27 (1973) 1837.

11. Cruickshank, D. W. J. Acta Crystallogr. 9 (1956) 754.

12. Schomaker, V. and Trueblood, K. N. Acta Crystallogr. B 24 (1968) 63.

13. Johnson, C. K. ORTEP, Report ORNL. 3794, Oak Ridge National Laboratory, Oak Ridge 1965.

14. Bernstein, J., Cohen, M. D. and Leiserowitz, L. In Patai, S., Ed., The Chemistry of the Quinoid Compounds, Wiley, London 1974, Part 1, Chapter 2.

15. Pauling, L. Nature of the Chemical Bond, Cornell University Press, New York 1960, p. 260.

16. Koetzle, T. F., Hamilton, W. C. and Parthasarathy, R. Acta Crystallogr. B 28 (1972) 2083.

17. Shimanouchi, H. and Sasada, Y. Acta Crystallogr. B 29 (1973) 81 .

18. Andersen, A. M. Acta Chem. Scand. B 29 (1975) 871, and references therein.

Received June 24, 1976.

Acta Chem. Scand. B 31 (1977) No. 1 